\title{
Matérialité linguistique des controverses sociétales. Rapports intersubjectifs et interdiscursifs dans des tweets polémiques
}

\author{
Agata Jackiewicz \\ Université Paris-Sorbonne, STIH \\ Agata.Jackiewicz@paris-sorbonne.fr
}

\begin{abstract}
Résumé. L'article s'intéresse à la matérialité linguistique des controverses sociétales. La démarche générale cherche à renouveler l'étude des controverses en intégrant les récents acquis des sciences sociales et politiques, en appui sur des corpus numériques issus des réseaux sociaux. Deux modes d'exploration des corpus sont proposés. Le premier est fondé sur les notions qui renvoient aux aspects caractéristiques des controverses (entités ciblées dans le débat, identité, postures et motivations des protagonistes, modes de validation critique des positionnements adoptés). Le second est guidé par des procédés linguistiques caractéristiques du registre polémique. La communication s'attachera à décrire, à caractériser et à systématiser les différentes formes d'interaction et d'emprunt à l'œuvre dans des tweets émis en contexte polémique.
\end{abstract}

\begin{abstract}
The article focuses on the linguistic materiality of social controversies. The general approach aims to renew the study of controversies by incorporating recent advances in social and political sciences, in support of digital corpora from social networks. Two ways of corpora exploration are proposed. The first one is based on concepts that refer to characteristic aspects of controversy (diversity of targets, protagonist's identity, positions and motivations, critical methods validating supported positions). The second one is guided by linguistic proceedings and markers characteristic of controversy register. Our communication describes, characterizes and systematizes different interaction's forms in tweets posted in polemical contexts.
\end{abstract}

\section{Introduction}

L’observation des productions verbales qui naissent et circulent sur les réseaux sociaux offre une fenêtre inédite sur les préoccupations et les métamorphoses affectant la société d'aujourd’hui. Il est possible étudier ces métamorphoses selon différents angles. L'un d'entre eux propose d'entrer dans l'analyse du monde social par ses moments critiques, en privilégiant la question des processus conflictuels.

Notre projet vise à construire des outils d'analyse et des ressources permettant de rendre compte des multiples formes d'interaction verbale en situation de polémique ou de controverse (Jackiewicz à paraître). L'objectif recherché n'est pas de produire des analyses de fond d'une controverse particulière, mais de proposer des outils linguistiques appropriés qui permettraient d'assister un tel travail, quel que soit l'objet et le contexte de la controverse. En vue des applications potentielles, il s'agit notamment de définir des schémas d'expression caractéristiques (mini grammaires, faisceaux d'indices robustes...), dont la coprésence dans des configurations particulières permettrait de révéler ou de confirmer la présence d'une controverse dans un corpus textuel.

L’étude du marquage linguistique des discours polémiques n’est pas nouvelle en sciences du langage. Tout en bénéficiant des résultats acquis, l’objectif est de renouveler la méthodologie d'analyse en intégrant les récents apports des sciences sociales et politiques, en appui sur des corpus numériques issus des réseaux sociaux. 
L'observation de ces nouveaux corpus nous amène à abandonner l'approche « classique " des controverses, laquelle met le focus sur la dichotomisation des points de vue et l'axiologisation des contenus. Même s'il s'agit d'un sujet fortement clivant, l'espace d'expression créé autour ne se réduit pas à deux pôles autour desquels gravitent des opinions « mono cibles », tranchées et univoques. Des attitudes ambivalentes, prudentes, distanciées ou neutres ne sont pas à exclure. L'enjeu est donc d'aller au-delà des approches dichotomiques, pour pouvoir appréhender des situations polémiques dans leur complexité inhérente, en tenant compte de la diversité des cibles, des attitudes et des points d'achoppement possibles.

Les observations que nous livrons dans les pages qui suivent concernent en priorité les usages polémiques sur Twitter. Nous considérons les corpus de tweets comme un laboratoire offrant accès à un matériau linguistique vivant et particulièrement riche. Sans oublier que la forme courte des tweets et les pratiques d'usage du dispositif influent sur les choix linguistiques pratiqués (Jackiewicz et Vidak 2014 ; Paveau 2015), nous voyons les énoncés qui y circulent comme des modèles élémentaires des verbalisations qui peuvent composer des discours polémiques plus élaborés.

La communication s’attachera à décrire, à caractériser et à systématiser les différents modes d'interaction et d'emprunt à l'œuvre dans des tweets à vocation polémique. La perspective que nous adoptons est celle de la sémantique discursive, que nous conjuguons avec des techniques d'analyse plus formelle (détection de n-grams, définition de patrons linguistiques, statistiques...).

\section{Cadre et contexte scientifique}

\subsection{Apports de l'analyse de discours}

La question des propriétés caractéristiques et des modalités verbales de la délibération polémique a débouché sur de nombreux travaux en analyse de discours. Signalons tout d'abord les apports majeurs de Kerbrat-Orecchioni (1980, 2005), Angenot (1982, 2008), Maingueneau (1983), ainsi les recherches de Amossy et de ses collaborateurs (Amossy et Burger 2011 ; Koren 2011 ; Amossy 2014). Des études plus ciblées, portant sur certains aspects des échanges agoniques, comme la violence verbale (insulte...), la rationalité et l'irrationalité ou l'implication émotionnelle, sont également éclairantes (Plantin 1997, 2011 ; Desmons et Paveau 2008 ; Moise et al. 2008 ; Lagorgette 2012, Oger 2012 ; Rosier 2012...). La discussion de la spécificité du débat polémique par rapport au débat argumentatif en général, entreprise notamment par Plantin (2003), constitue une autre technique pour faire ressortir ses traits les plus saillants.

Sans aboutir à un consensus, les auteurs (ceux cités plus haut, mais également Dascal 1998 ; Dascal \& Chang 2007 ; Rennes 2007 ; et bien d'autres) proposent différentes configurations de traits, nombreux et relatifs à des réalités variées. En voici ici les principaux, rassemblés dans une liste simple, en respectant la formulation d'origine (d'où l'inévitable recoupement entre des expressions citées) :

- polarisation et dichotomisation des thèses antagonistes,

- $\quad$ permanence de la question, stabilité relative des positions et des argumentaires,

- $\quad$ relative persistance de la différence d'opinion, désaccord durable,

- caractère recyclable (mémoire polémique),

- désaccord sur des questions ayant des enjeux (religieux, politiques, idéologiques) importants,

- font l'objet de polémiques les débats entre visions du monde radicalement incompatibles,

- mobilisation publique de deux camps emmenés par les polémistes en vedette,

- désaccord fondamental, dissensions profondes,

- $\quad$ existence d'un « nœud d'arguments contradictoires récurrents »,

- présence d'un proposant, d’un opposant et d'un tiers,

- confrontation visant à discréditer l'adversaire et à emporter l'adhésion du tiers,

- tentative de falsifier la parole de l'autre,

- $\quad$ présence significative de paralogismes, mauvaise foi, « dialogue de sourds ",

- $\quad$ engagement émotionnel (spécialement dans l'évaluation dépréciative), 
- $\quad$ différentes formes de violence « ordinaire » (agressivité...),

- $\quad$ sorte de guerre verbale ${ }^{1}$,

- $\quad$ mise en jeu d'une identité fondée sur des valeurs de groupe...

A l'évidence, la notion de polémique renvoie à un phénomène socio-langagier multidimensionnel, dont les propriétés définitoires relèvent de différents ordres (social, politique, psychologique, linguistique, discursif...).

De même, on a cherché à répertorier les procédés caractéristiques des discours polémiques. Ont été notés entre autres : (i) différents phénomènes d'énonciation localisés (apostrophes, formules phatiques,...), (ii) usage massif du discours rapporté, (iii) emploi des axiologiques, des modalisateurs, (iv) termes d’émotion, (v) certaines techniques argumentatives (ad hominem...), (vi) formes hyperboliques ...

\subsection{Projet et démarche}

Notre projet global vise l'élaboration d'outils d'analyse et de ressources permettant de rendre compte des multiples formes d'interaction verbale manifestant des procédures de négociation des points de vue divergents ou antagonistes.

Partant d'une sélection de travaux de référence en sociologie des conflits, sciences politiques et sciences d'information et de communication (Latour 2007 ; Lemieux 2007 ; Chateauraynaud 2011 ; Reynié 2012 ; Julliard et Cervulle 2013 ; Cervulle et Pailler 2014), nous avons élaboré une grille d'analyse dont les notions constitutives renvoient à différentes facettes de la conflictualité (Jackiewicz à paraître). L'idée que nous défendons est que le profilage notionnel des productions langagières collectées, souvent foisonnantes, riches et bruitées, permet d'ordonner analytiquement le matériau discursif associé à une controverse.

Dans un deuxième temps, chaque notion du modèle a été caractérisée par un champ lexical caractéristique qui forme sa signature lexicale. La projection des lexiques sur le corpus FILIATION (voir §2.4) a permis de sélectionner les ensembles de tweets correspondant à chacune des dimensions de la controverse (corpus profilé). A partir de ces données, nous avons pu apprécier la présence dans le corpus et la rentabilité des lexiques originels. Cette opération a permis de s'assurer que l'ensemble des dimensions du modèle sont représentées dans le corpus d'observation, ce qui confirme que le corpus renferme bien des productions verbales émises en situation de controverse. Nous avons réitéré ce traitement sur le corpus MARIAGE avec des résultats sensiblement comparables. L'analyse linguistique des tweets ainsi catégorisés a conduit à l’identification des schémas d'expression caractéristiques, ainsi que des procédés discursifs (argumentatifs, énonciatifs, rhétoriques..) impliqués dans le marquage des énoncés de nature polémique.

Dans un troisième temps, nous avons étendu et systématisé le répertoire des procédés linguistiques pertinents par un va-et-vient entre le corpus et des études linguistiques spécifiques portant sur des phénomènes identifiés dans le corpus (négation polémique, reformulation polémique, termes d’adresse etc.).

\subsection{Deux modes d'exploration et d'analyse}

Au final, deux modes d'exploration des corpus sont proposés. Le premier est fondé sur les notions qui renvoient aux aspects caractéristiques des controverses. Le second est guidé par les procédés linguistiques remarquables.

La grille notionnelle structure un ensemble de catégories, de paradigmes et de notions qui renvoient à la nature des entités ciblées dans le débat, aux postures et aux motivations des protagonistes, ainsi qu'aux modes de validation critique des positionnements adoptés. On focalise ici l'attention sur ce qui constitue l'essence même d'un processus polémique propre à une controverse. Les procédés linguistiques, quant à eux, se répartissent entre cinq grandes catégories qui correspondent aux stratégies argumentatives, 
relations idéationnelles, rapports intersubjectifs, formes d'interdiscursivité et marques lexicales. Ces différents procédés ne sont pas exclusifs entre eux. Au contraire, souvent ils s’associent ou s’imbriquent en discours.

Chacun de ces modes d'exploration offre des lectures particulières du matériau verbal associé à une controverse. En fonction de que l'on recherche on pourra se laisser guider par tel ou tel procédé linguistique, telle ou telle notion, ou enfin par telle ou telle association de procédés linguistiques et de notions. A terme, il s'agira d'outiller cette méthode, sous forme d'une plateforme d'annotation et d'analyse, pour pouvoir notamment articuler les deux modèles. Dès lors, il sera possible de l'éprouver sur des controverses de différents types, tester, préciser voire enrichir les facettes, les procédés et les ressources linguistiques.

\subsection{Corpus}

Notre corpus d'étude est constitué de deux collections de tweets en rapport avec un ensemble de sujets polémiques touchant au mariage, à la procréation et à la filiation. Ces questions sont de nature à trouver un très large écho dans la société civile et donc sur l'ensemble des dispositifs de communication qu'elle emploie. Le premier corpus porte sur l'ouverture du mariage aux couples de personnes de même sexe (corpus MARIAGE, noté MAR). Le deuxième concerne la question de la filiation issue de la gestation pour autrui (GPA) (corpus FILIATION, noté FIL). Les tweets sont datés pour permettre d'adosser l'analyse aux événements qui ont constitué les temps forts de ces deux controverses (débats, auditions, manifestations nationales...).

Le corpus FIL, notre corpus principal, a été collecté automatiquement le 5 octobre 2014, durant 24 heures, à partir des mots clés suivants : pma, gpa, procréation pour autrui, gestation pour autrui, filiation, famille, manif pour tous, manifpourtous. Il comprend au total 184300 tweets. En simplifiant, la mobilisation organisée ce jour servait à montrer l'opposition à la gestation pour autrui (GPA) et installer dans l'espace public la cause d'une filiation « naturelle », vécue comme menacée par le récent accès au mariage accordé aux couples homosexuels.

Bien que volumineux, ces corpus d'observables sont nécessairement incomplets. En effet, les contenus extraits de Twitter ne représentent pas des ensembles clos, complets et cohérents de significations, et cela quel que soit le mode de collecte déployé. C'est une portion d'un vaste interdiscours qui renvoie à un espace écologique complexe (tweets, retweets, contenus connexes accessibles via des liens URL...). Cet espace fait à son tour partie d'une configuration plus vaste qui associe étroitement presse imprimée et en ligne, radio, télévision, Internet...

\section{Discours éristiques sur Twitter}

Les nouveaux dispositifs de communication numériques offrent des espaces d'expression et d'échange, massivement investis par la société. Ces espaces médiatiques en ligne articulent différents niveaux d'interaction : de l'individu à ses cercles sociaux, du privé au public, de l'objet à ses référencements, reprises et commentaires. Ils induisent de nouvelles formes verbales et de nouveaux genres textuels, souvent complexes et multiformes. Chaque production écrite ou orale (tweet, message sur Facebook, billet de blog, article de presse en ligne, vidéo...) est potentiellement commentée ou évaluée, citée ou référencée, reprise en l'état, enrichie ou détournée.

Le dispositif communicationnel et énonciatif de Twitter produit des conditions spécifiques d'échange polémique, différents de ceux qui sont habituellement analysés - débats ou duels télévisés, séries de discours monogérés compilés et rapportés par des médias - (Amossy 2014). Dans la sphère du Web, les échanges polémiques sur des forums de discussion ou des pages de commentaires associés à des articles, se rapprochent des tweets par leur aspect polylogue et le caractère anonyme des participants, mais s'en s'éloignent par plusieurs points propres aux tweets comme: l'absence d'ancrage, l'absence de modération, l'ampleur de la participation, la forme courte, les pratiques d'adressage et de reprise massive. 
Si Twitter peut être vu comme un lieu de confrontation des opinions, le mode d'interaction induit par son dispositif favorise en réalité une multiplicité de contributions et de micro-débats. Souvent les opinions évoluent en parallèle, plutôt qu'elles ne se confrontent véritablement.

Chaque tweet est porteur d'un message court qui représente - au mieux - une étape dans l' «itinéraire $\operatorname{argumentatif}^{2}$ » du locuteur. Il évolue parmi des milliers d'autres tweets, dont il rencontre ou croise certains grâce au système d'adressage, les mots-dièse et les liens hypertexte. On a donc affaire à une constellation verbale, a priori impossible à délimiter, car prise dans une dynamique mouvante et incessante des flux médiatiques et des interactions. C’est une narration fragmentée du monde, faite de manques et d'incohérences, mais actualisée en permanence. On peut néanmoins espérer pouvoir y trouver une forme de pensée collective, développée à plusieurs voix, laquelle mérite, à nos yeux, d’être considérée et interrogée.

Les discours éristiques sur Twitter se distinguent par plusieurs caractéristiques remarquables. La forme courte du tweet conduit les internautes à dire l'essentiel en peu de mots. De ce fait, les verbalisations prennent une forme directe et synthétique. Les opinions défendues et les arguments avancés sont souvent exprimés dans des schémas d'expression simples et stéréotypés. Les débatteurs emploient des mots-dièse pour marquer le thème (\#GPA, \#maternité, \#mariagehomo...), cela est habituel, mais aussi pour appuyer leur position (\#ProMariageGay, \#ContreLaGPA...) ou signaler que leur propos revêt un caractère polémique (\#contradiction, \#délire, \#duel, \#crédibilité...).

1. Je suis \#ProMariageGay et je suis fière de mes convictions. \#LaManifPourTous \#ManifDeLaHonte

2. La \#Maternité, précieux élément du patrimoine de l'humanité : à ne pas occulter, escamoter ni éclater" \#GPA \#5oct

3. La \#manifcontreautrui dit "la femme n'est pas une usine à enfants". Ils sont donc pour l'avortement en fait ! \#contradiction \#manifpourtous

Les tweets émis en situation de polémique comportent fréquemment des marques interactionnelles et conversationnelles (termes d'adresse, interpellations, déictiques, marques d'opposition...), même s'il ne s'agit pas d'une interaction à proprement parler. L'évocation du destinataire (se confondant souvent avec l'adversaire discursif, mais pas systématiquement) peut aller de la désignation nominale, en passant par une spécification catégorielle ou une qualification, à l'allusion pure. De manière plus inattendue, on constate que de nombreux tweets comportent à leur initiale des connecteurs, notamment argumentatifs (parce que, mais, par contre, et sinon, oh oui alors, en vrai, en tout cas, du coup...). Cela révèle que les auteurs tendent à articuler leurs messages avec des discours autres, qu’ils soient réels (publiés sur Twitter ou ailleurs, lus ou entendus lors d'une manifestation...) ou fictifs.

4. Je pose la question aux partisans de la \#GPA : avez-vs un exemple d'une femme issue d'1 classe aisée faire la GPA pour un couple de pauvres?

5. Mais mobilisez-vous donc pour de vrais causes! Bande de nazes... \#ManifDeLaHonte

6. Nan mais les gens de la manif pour tous ils sont en 1960, ils s'apprêtent à élire Valérie Giscard d'Estain.

7. Parce que la famille n'a pas pour seul modèle \#ManifPourTous, un peu d'amour. \#TweetPourTous

8. Par contre il faudrait créer un statut particulier pour les gosses issus de GPA à l'étranger

9. Et apres on retournera a l'esclavagisme vous etonnez pas \#manifpourtous \#porteouverte

La forme courte du tweet pousse les contributeurs à des reprises de discours (phrases, formules, termes...) ayant une résonance sociale importante. Pour susciter l'adhésion, les opinions exprimées tendent à mobiliser des lieux communs, des topoï culturellement sédimentés, des formes socialement acceptables. Par ce moyen, le tweet gagne en épaisseur (dialogique) et en légitimité, en s’inscrivant ainsi dans un courant de pensée, une mouvance ou une tradition. Par ailleurs, le recours à des unités de discours ayant un pouvoir évocateur important, notamment historique (esclavage, ségrégation, politiques natalistes de Vichy, eugénisme, les \#SansDents...), permet d'orienter nettement le sens de l'argumentation, en s’appuyant sur la teneur et la charge axiologique des discours déjà tenus. 
10. "L'être humain n'est pas une marchandise".

11. il est raisonnablede lutter contre l'esclavage\& la marchandisationD êtres humains ss défense

12. Les "gays" se défendent en avec la ségrégation, un Homme ne choisit pas sa couleur de peau... manque de dignité de leurs part \#manifpourtous

13. GPA et PMA étaient l'état d'âme d'Adolf HITLER: sélection humaine, enfants parfaits, eugénisme, que de l'imbuvable

14. Donc, en fait, la \#manifpourtous, c'est juste à Vichy ou dans toute la France?

15. Les Bobos n'en peuvent plus, la famille serait une invention de Pétain !

Dans les sections qui suivent nous nous attacherons à détailler, caractériser et systématiser les manifestations linguistiques du fonctionnement interactionnel et interdiscursif des tweets polémiques.

\section{Rapports intersubjectifs}

L'activité polémique est indissociable des interactions interpersonnelles. De ce fait, l'ensemble des procédés énonciatifs qui sous-tendent des échanges verbaux entre locuteurs sont à considérer. «Etant un discours de passion, le discours polémique doit être explicitement assumé par son énonciateur ; étant un discours de persuasion, il est en même temps tourné vers son destinataire qu'il s'agit de convaincre, séduire ou circonvenir. Ce qui veut dire que ce type de discours partagerait avec quelques autres la propriété d'être très fortement marqué énonciativement », nous dit Kerbrat-Orecchioni (1980 : 16).

Les conditions spécifiques créées par le dispositif du réseau Twitter font qu'il ne s’agit pas d’un dialogue au sens classique du terme. Nous avons plutôt affaire à une somme de voix portées dans l'espace public. Elles sont singulières, nombreuses, chaotiques, et seulement certaines d'entre elles se répondent réellement. Mais à la lecture du corpus entier, force est de constater que la présence de déictiques, d'interpellations, de formules phatiques, de réponses à des questions rapportées crée un effet conversationnel certain.

\subsection{Déictiques, marques d'adresse, formules phatiques...}

L'habileté à argumenter et polémiquer se manifeste très nettement dans les façons d'interpeller et d'impliquer l'autre. Les déictiques personnelles, les marques d'adresse et les formules phatiques (ditesmoi, dis donc, tu penses ! eh ! ...) sont les traces privilégiées des débats et des affrontements verbaux. Celles-ci servent à établir entre les interlocuteurs un certain type de lien socio-affectif. Elles s'associent généralement avec la modalité interrogative ou injonctive.

Le recours aux termes d'adresse sur Twitter traduit très clairement le besoin d'interpeller celui qui sera souvent à la fois destinataire et cible du message. Cela tient au dispositif technolangagier employé. Le contexte polémique favorise très clairement ce type d'attitude ${ }^{3}$.

La prise à partie de l'adversaire conjugue souvent interpellation et désignation, notamment grâce à des noms d'adresse ou appellatifs (catégoriels : termes de profession, d'appartenance sociale ou religieuse... ; termes injurieux ou affectueux...). Cet acte peut prendre la forme d'une exclamation relationnelle agressive. L’insulte qui est une forme particulière d'adresse sera traitée dans le \&4.4.

16. Eh, les rétrogrades de La \#ManifDeLaHonte! C'est pas si dur la tolérance : moi je vous le laisse, votre droit constitutionnel d'être idiots

17. éh les homophobes, si un gosse a besoin de repère mixte, comment font les enfants issues d'une famille mono parentales? tsss idiots

18. Aux partisanes de la \#GPA je dis : montrez-nous l'exemple. Faites des enfants et offrez-les gratuitement aux couples stériles. Chiche?

19. Stop à la familiphobie! Socialistes, ça suffit ! Stop à votre idéologie! Non à la deconstruction de la famille! \#lmpt

20. Et puis honnêtement, vous leur apprenez quoi à vos enfants en les emmenant dans cette \#manifdelahonte ?" L'intolérance ? L'irrespect ? Oui ! 
21. Dites moi, est-ce qu'une fois on a entendu parler de couple homosexuel qui tue leur enfants ? Non. La réponse à tout. \#ManifDeLaHonte

22. Putain mais $\boldsymbol{j}$ 'vous comprend pas a être contre le mariage pour tous. Enfaite, allez bien vous faire foutre !! \#ManifDeLaHonte

\subsection{Modalités de prise en charge énonciative}

La prise en charge énonciative est typique de l'expression des opinions. Elle dévoile les modes d'adhésion et de distanciation par rapport aux états des choses soumis au débat. La prise en charge sert en particulier à transmettre une conviction, ce qui constitue l'un des objectifs principaux de l'argumentation. Ses modalités explicites sont souvent portées par des verbes d'attitude propositionnelle employés à la première personne.

23. Je suis \#ProMariageGay et je suis fière de mes convictions.\#LaManifPourTous \#ManifDeLaHonte

24. Je comprends tout à fait qu'on puisse être contre le mariage pour tous ou dubitatif sur la question de la PMA/GPA.

25. Je suis vraiment pas d'accord avec cette manif.. Je rappelle que dans notre devise il y a " Égalité " \#TF1 \#ManifDeLaHonte \#ManifPourTous

26. Je suis en désaccord profond avec les élus @ump présent à la \#manifpourtous. Faire croire à l'abrogation de la loi Taubira est un mensonge.

Des prises de position plus nuancées, signalées par des connecteurs contre-argumentatifs (mais...), méritent d'être identifiées et traitées à part, dans la mesure où elles échappent à l'apparente binarité du débat. Par exemple, on peut dissocier nettement la question du mariage et celle de la procréation. On peut s’avouer opposé à la GPA et la PMA, tout en prenant ses distances avec le collectif Manifpourtous.

27. Je suis contre la \#GPA et je suis pour le mariage pour tous. Je ne comprends pas les gens qui mélangent les deux!

28. Je rejoins @alainjuppe sur le \#mariagepourtous. Personne ne pourra revenir s/ le mariage homosexuel mais interdisons clairement \#GPA et \#PMA.

29. Je suis contre la PMA et GPA, mais je ne pouvais pas manifester auprès de ceux qui refusaient aux homosexuels, le mariage. \#ManifPourTous

30. Je précise que je ne suis pas pour la GPA, mais passer son temps a empêcher les gens d'être heureux, c'est nul et il faut reconnaître $1 / 2$

31. Je suis contre la GPA et PMA du \#mariagepourtous, mais je crois plus urgent de se mobiliser contre la CRISE qui ns détruit TOUS. C'est clair?

Des cas de détachement ironique traduisant une distance ou un désenchantement sont à également à considérer. Dans l'ironie, il y a un effet 'non-prise en charge' de l'énonciation par le locuteur et discordance par rapport à la parole attendue dans tel type de situation. En général, l’ironie est employée pour dévaloriser. "Ironiser, c'est toujours plus ou moins s'en prendre à une cible qu'il s'agit de disqualifier » (Kerbrat-Orecchioni 1986 : 102).

32. Je comprends la présence de MAM au défilé. Elle sait la force et l'importance d'une famille soudée comme celle de Ben Ali par exemple

\subsection{Expression des émotions sociales}

Dans les interactions sociales la parole entretient des rapports multiples et fréquents avec l'émotion. Rappelons qu'au sens de Scherer (1984), l'émotion correspond à un éprouvé subjectif ayant des manifestations corporelles végétatives et des manifestations comportementales observables (expressives, réactives). Si l'émotion affichée ou perceptible fait partie du sens communiqué, celle qui est explicitement verbalisée en est indissociable. 
L'expression des émotions intervient dans la régulation des interactions humaines (ajustements interindividuels, coordination avec autrui...) et dans la construction des relations sociales. Le partage social des émotions peut en effet instaurer un rapprochement affectif entre des personnes qui ne possèdent pas de liens préalables. Une fonction des émotions serait de créer, maintenir ou renforcer les liens sociaux par des mécanismes d'empathie. Les expressions émotionnelles d'un individu fournissent également des informations sur ses états mentaux (intentions, sentiments, croyances, désirs), qu'il s'agisse d'une posture contrôlée de dévoilement de soi, ou d’une "décharge émotionnelle" verbale (le cas typique en est la décharge expressive souvent incoercible de la colère).

On le sait, les utilisateurs de Twitter n’hésitent pas à verbaliser leur ressenti et à le rendre public. Les réactions émotionnelles présentes dans nos corpus sont très variées : spontanées ou produites en réponse à une invitation explicite, communiquant son propre ressenti, spéculant sur l'état émotionnel de l'adversaire ou répondant à des sentiments que ce dernier a choisi d'afficher, se positionnant en tant qu'acteur du débat ou seulement comme un témoin extérieur. Un vaste spectre d'émotions négatives (colère, honte, indignation, exaspération, agacement, mécontentement...) est clairement perceptible dans le corpus FIL.

33. \#ManifDeLaHonte J'ai de la colère, du dégoût, de la peine. Mais j'ai de l'espoir aussi, espoir qu'un jour tout se retourne contre vous!

34. C'est la GPA qui est une honte ! Elle fait de l'enfant un produit et le corps de la femme est commercialiser ça oui c'est une HONTE!

L'expression directe des émotions sur Twitter est souvent stéréotypée. Voici quelques schémas d'expression récurrents de nature relationnelle traduisant une réaction émotionnelle face aux idées (agissements...) du camp adverse. Comme le montrent les tweets cités, il serait vain d’y chercher une quelconque trace d'éléments factuels du débat.

«Vous me + SV+ avec + votre/vos/... + SN » : vous me faites chier/ donnez la nausée/les brisez/m'petez les couilles/soulez/gonflez/dégoutez ...+ ...

35. Vous me donnez la nausée avec votre \#ManifDeLaHonte

36. Vous me faites $c^{* * * *}$ avec votre \#ManifPourtous... Franchement STOP!!

37. Vous me les brisez avec votre manif pour tous. Laissez les gens vivre, merde!

38. Vous m'petez les couilles avec la Manif pour tous Laissez ces abrutis manifestaient tant qu'ils le veulent ils en ont le droit

«ça me V + SN » : Ça me dégoute/dérange/horripile/inquiète/énerve/rend folle, malade.../écœure/tue/ gave/ gonfle/soule/ révolte/fout la gerbe, la honte, les nerfs .../donne la nausée, envie de rire... +...

39. Ça me rend dingue la manif pour tous, j'pourrais devenir homo juste pour les faire chier.

40. ca me fait rire les anti \#ManifPourTous donneurs de leçons avec des propos tout nauséabonds

41. Ça me fait rire les mecs qui militent dans la \#ManifPourTous alors qu'ils se branlent sur du porno lesbien. \#ManifDeLaHonte

« J'ai (tellement /très/vraiment...) envie de + V + (quand/en...) » : j’ai envie de pleurer / gifler / vomir /vous cracher ma haine / twitter plein de conneries/ ...

42. J'ai envie de pleurer quand je vois les opinions sur la manif pour tous. Des deux côtés. \#Manifdelahonte

43. J'ai envie de vomir en vous voyant tous défiler putain mais vous êtes inhumains. Je vous crache à la gueule. \#manifpourtous \#Manifdelahonte

44. J'ai envie de gifler un par un les frustrés-anti progressistes de la manif pour tous

Dans le corpus FIL, le sentiment de honte est fréquemment évoqué, et cela tant par les deux camps opposés que par des observateurs. Le détournement de la désignation Manifpourtous en ManifdelaHonte par les adversaires de ce collectif en est la trace la plus saillante. L'expression de ce sentiment peut venir accompagner en le renforçant l'acte de réfutation de la position de l'adversaire. La honte, comme la colère, sont souvent présentes dans des messages qui dénoncent l’inacceptable. 
45. "La France a besoin d'enfants, pas d'homosexuels" Omg j'ai honte. \#ManifDeLaHonte

46. "Je ne veux pas que ma mère s'appelle Robert" HONTEUX! \#ManifDeLaHonte

L'expression de l'émotion à travers les mots-dièse n'est pas rare. C'est une forme à la fois saillante et brève d'imprimer un jugement de valeur au contenu ainsi ciblé. Dans le corpus FIL, on identifie 83 motsdièse d'émotion différents, négatifs pour 81\% d'entre eux, dont \#honte, \#tristesse, \#dégoût, \#désespoir, \#colère, \#déception... Le caractère saillant d'une émotion est révélé par des créations à partir d'un même terme, augmentant ainsi son épaisseur sémantique. Par exemple, pour la honte et la tristesse nous avons respectivement (i) \#honteacesesclavagistes, \#honteaeux, \#hontepoureux, \#honteàvous, \#honteavousplutot, \#hontedelafrance, \#hontedetrefrancaise... (ii) \#tristessepourtous, \#TristeRealite, \#TristeFrance, \#tristepays...

Des combinaisons de mots-dièse d'émotion - généralement de même polarité - sont également à signaler [\#haine, \#homophobes], [\#haine, \#menaces], [\#joie, \#allegresse], [\#PeurDeSeMouiller, \#lachete], [\#Tristesse, \#HontePourEux], [\#TristessePourTous, \#ManifPourTous]. On peut y trouver également des mots d'insulte.

\subsection{De l'humour à l'insulte}

Les figures d'humour, d'ironie ou de sarcasme sont des armes fréquemment employées pour écorner l'image de son adversaire, ridiculiser ses idées, mettre en doute sa crédibilité. Depuis des mots gentiment moqueurs ou des « vannes » jusqu'aux formes les plus brutales de la ridiculisation, la palette des moyens disponibles est aussi vaste qu'impossible à ordonner (sur une hypothétique échelle de nuisance ou d'agressivité). Certains messages humoristiques se révèlent en réalité d'une violence verbale extrême. Un brillant trait d'esprit peut être décoché pour humilier ou nuire.

Si dans toute argumentation il y a confrontation, on peut soutenir que la violence verbale dirigée vers l'autre est aux prémices de toute situation d'argumentation, comme un danger potentiel selon les enjeux, les rapports entre les protagonistes et leur implication émotionnelle face aux éléments du débat. En particulier, quand un combat social et moral se double d'un combat politique (comme c'est le cas pour le mariage et la filiation), la disqualification de l'adversaire, que l'on voit si souvent à l'œuvre en politique, devient une règle de jeu quasiment obligatoire.

Intégrée dans un processus polémique, l’insulte constitue une véritable arme dirigée contre l'adversaire : pour lui «clouer le bec », pour toucher son point sensible (faire mouche), l'éjecter en dehors de la discussion... Mais l'usage de l'insulte a également d'autres fonctions. Certaines insultes semblent rechercher un effet défoulant, d'autres témoignent d'une irritation et d'une exaspération. Les insultes jouent généralement sur un double tableau de l'émotion et de l'argumentation. Rosier (2012) signale quant à elle « sa dimension rituelle et la mise en scène des affects sociaux qu'elle permet ».

Si Twitter peut être vu comme source d'une parole authentique et « saine » (spontanée, insolente, drôle, peu policée), en rupture avec le politiquement correct et avec la politesse verbale imposée par les conventions sociales, c'est aussi une arène de l'outrance langagière et de l'agressivité. Le mode de fonctionnement de ce dispositif, par l'anonymat de ses utilisateurs et l'absence de modération, permet (favorise ?) la désinhibition et les débordements de toute sorte. Des pratiques déviantes, voire violentes seraient elles moins graves aux yeux des gens, dès lors qu'elles se déroulent dans un espace virtuel ?

Comme il est très difficile d'isoler l'insulte en tant qu'acte dans un discours (Laforest et Vincent 2004), nous nous intéresserons ici à un ensemble de formes de qualification péjorative (moquerie, vanne, dénigrement, outrage, diffamation...), sans tenter d’opposer formellement ou sémantiquement ces usages.

Dans le corpus analysé, des références à la religion et au passé, via la métaphore du Moyen âge notamment, ont été massivement exploitées pour dénoncer les idées rétrogrades des membres du collectif Manif pour tous. Des mots-dièse spécifiques ont été forgés à cet effet: \#retouraumoyenâge, \#TeamMoyenAge, \#teamXIIemesiècle, \#teamcoincésducul, \#TeamHyperconservateurs, \#TeamLigonnès, \#TeamdeVilliers... 
47. Avant ils brûlaient les sorcières, maintenant... \#ManifDeLaHonte \#LaManifPourTous \#LaManifdelahonte \#retouraumoyenâge

48. "Abolition universelle de la GPA" carrément ... \#teamXIIemesiècle

49. Avant ils multipliaient les pain, maintenant c'est les manifestants ... \#Magique ! \#ManifDeLaHonte

50. Le preux chevalier et sa gente dame partirent promptement à la manif pour tous

51. Tiens des gens et des caméras dans les rues de Paris? Qu'est ce qui se passe? Le tournage de 'Les Visiteurs 4' ?\#LMPT \#ManifDeLaHonte

52. Sympa cette procession médiévale, très authentique \#ManifPourTous

Les adversaires désignés à la vindicte voient leurs noms accompagnés de descriptions ou qualificatifs injurieux, leur capacité mentale déniée, leurs pratiques (religieuses...) ridiculisées, leurs (prétendues) tares physiques soulignées. L’insulte prétend rendre compte de la spécificité condamnable de l’autre.

53. Entre l'instrumentalisation de leurs gosses, leur étroitesse d'esprit et leur haine, ils nous emmerdent les cons de la \#ManifDeLaHonte!

54. De fervents catholiques égrainent des chapelets en récitant un "Je vous salue Marie" à la \#ManifPourTous

55. \#gpa \#13h15 Ces deux pédales n'ont pas à revenir en France! Il faut qu'ils restent la bas. Et l'autre, un long nez, des grandes oreilles..

Certains formats d'insultes, comme 'bande de X', sont adaptables au contexte et à la cible visée (d'esclavagistes, d'arriérés rétrogrades, de connard étroit d'esprit, d'égoïstes intolérants ridicules,...). On note par ailleurs des insultes usuelles (con, connard, débile, attardé, crétin, abruti, FDP, boloss, trous du cul...), communautaires (nique tes morts), des tours classiques (sale $\mathrm{X}$, petit $\mathrm{X}$, tas de $\mathrm{X} . .$. ), etc. On constate aussi une tendance à former des mots-dièse insultants, à partir d'un amalgame : appelatif + mot d’insulte (\#Gauchebarrée, \#gauchecaviar, \#gauchenazie, \#gauchetarée...).

56. Sérieux, l'erreur est d'avoir voulu comprendre les débiles de manifpourtous, maintenant faut remettre ces tas de merdes à leur place

57. Que des petits cathos ou des gens de droite et d extrême droite.... \#ManifPourTous \#ManifDeLaHonte

58. Les bobos de \#gauche nous ressortent leur défense pitoyable... Sale fachos... la \#ManifPourTous Comme d'habitude...

Les insultes directes (adressées et performatives) sont caractéristiques des tweets agressifs, ne présentant pas (ou ne présentant que peu) d’éléments factuels en lien avec le sujet du débat.

59. Eh les homophobes vous êtes cons, débiles, pathétiques vous êtes juste une bande de connards sans cerveaux \#ManifDeLaHonte

60. Arrêtez d'endoctriner des enfants dans vos sales délires bande d'attardés mentaux ! \#manifdelahonte \#déséquilibrés

61. dédicace à toutes les chiennes de Satan présentes à la \#ManifDeLaHonte et qui mêlent leur enfants à ça \#ManifPourTous http:

62. Bande d'arriérés rétrogrades. Le monde change, changez avec et arrêter de vivre au Moyen-Âge ! \#ManifPourTous \#ManifDeLaHonte

La frontière entre description, insulte et allégation diffamatoire est parfois difficile à établir. De même, on peut signaler quelques tweets lesquels pris au pied de la lettre expriment une menace ou un appel à la haine. L’interprétation de tels tweets reste délicate.

63. Aucune leçon à recevoir de cette gauche en tête de toutes les manifs haineuses, de casseurs, où on crie "mort aux juifs". \#manifpourtous

64. Tous ces \#gaucho qui haïssent nos anciens et qui préfèrent la sodomie.. \#triste \#ManifPourTous

65. Qui achètent des enfants par GPA ? Les blancs! \#GaucheBarrée

66. \#LesPédésAuBûcher 
67. \#ManifDeLaHonte mort au fachos.

68. La bétaillère à catho qui se gare en bas de chez moi pour aller à la manif pour tous, je vais les lapider.

\section{Interdiscursivité et dialogisme}

Dans une interaction polémique, discours et contre-discours s'articulent au moyen des stratégies de reprises discursives, réinterprétations, concessions, réfutations, négations... Des fragments de discours autres - sous forme de référence, citation, allusion, reformulation, négation, détournement...- sont potentiellement présents dans chacune des interventions constituantes.

\subsection{La citation}

L’usage de la citation - qui renvoie au sens large aux différents modes de représentation des discours autres - peut servir des finalités multiples : épouser un point de vue, le combattre, le subvertir, louer ou discréditer celui qui en est porteur... Ces différentes stratégies de réinvestissement d'un discours, au sens de Charaudeau et Maingueneau (2002 : 93), dépendent étroitement du genre de discours et du positionnement du locuteur, en fonction de son identité sociale et idéologique.

En situation de controverse, les discours respectifs sont repris et interprétés au cours de l'interaction verbale et souvent "adaptés » en fonction des besoins des interlocuteurs. La circulation des propos polémiques est génératrice de commentaires négatifs, de transformations importantes, voire d'oublis volontaires, l'objectif étant de falsifier la parole de l'autre, afin de la disqualifier. Parmi les schémas discursifs récurrents nous trouvons la réfutation. Il s'agit un acte réactif argumentatif d'opposition, qui suppose une reprise du discours via certains mots au moins, ou une connexion avec ce discours. Son objectif radical est la destruction du discours attaqué. Ses modalités possibles sont nombreuses: disqualification du discours en raison de sa malformation, rejet d'un type d'argument (d'autorité, de comparaison...), disqualification ciblant l'adversaire lui-même, par exemple, en raison d'une contradiction entre ses actes et ses paroles.

Dans le corpus «FIL », les tweets de citation et les retweets à l'identique (ou presque) sont un moyen simple de signaler l'adhésion à une parole jugée pertinente, tout en en augmentant l'audience et l'impact. Certains tweets détaillent l'origine ou le parcours d'une citation.

69. On entend "GPA esclavage, Hollande dégage !" \#LMPT \#5octRN

70. @Maitre_Eolas: En tant que catholique, je suis pour la GPA puisque c'est comme ça que Jésus est né.

71. "On instrumentalise la famille pour promouvoir un modèle religieux" @benoithamon à propos de@LaManifPourToussur@franceinter.Bien dit!

72. " sur la \#GPA je salue la position de Manuel \#Valls " @christineboutin sur @sudradio invitée politique 8 h10

73. "la haine est la colère des faibles" A. Daudet \#ManifDeLaHonte

Le désaccord, quant à lui, a besoin d'un signalement explicite. Celui-ci peut prendre la forme d'un commentaire minimal (mot-dièse modal...) ou passer par une réaction plus élaborée.

74. "Je ne veux pas que ma fille loue son ventre si elle a des problèmes d'argent !". Et si elle loue son cul ça te choque moins? \#GPA \#BFMTV

75. "La non-discrimination n'est pas l'indifférenciation" Fou comme les convictions se périment vite \#ManifPourTous \#5oct

76. "La famille c'est un père et une mère" Dans ce cas là, allez enlever les enfants qui n'ont plus qu'un père ou une mère, ça serait logique.

77. C'est une vision tlmnt chrétienne des choses, "une famille c'est un homme une femme et des gosses". On est en 2014 il serait temps d'évoluer

78. "On va créer des dégénérés"... C'est pas vous les dégénérés plutôt ! \#ManifDeLaHonte 
De nombreux tweets sont postés en réaction à une affirmation ou à un slogan, entendus lors de la manifestation ou dans les médias. Une négation polémique peut être reprise et attaquée à son tour, comme dans 85-86.

79. "La France a besoin d'enfants, pas d'homosexuels" Omg j'ai honte. \#ManifDeLaHonte

80. "La France a besoin d'enfants, pas d'homosexuels" et les enfants gays ? les homos qui veulent des enfants ?!\#ManifDeLaHonte \#ManifPourTous

81. "la france a besoin d'enfant pas d'homo" qui vous dit que les enfant sont contre les gays? \#ManifDeLaHonte

82. "La France a besoin d'enfants, pas d'homosexuels" Lol. Y'a même pas de boulot pour les enfants de la France... \#ManifPourTous \#Chomage

83. "La France a besoin d'enfants, pas d'homosexuels" NON LA FRANCE N'A SURTOUT PAS BESOIN DE FDP COMME VOUS. \#ManifDeLaHonte

84. "LA FRANCE A BESOIN D'HUMAIN PAS D'HOMOPHOBES" c'est une bonne réponse a leur banderole \#ManifDeLaHonte

85. "La femme n'est pas une machine à bébés", dit cette militante de la \#ManifPourTous mère de 5 enfants.

86. "La femme n'est pas une usine à bébé!". Dixit une femme interviewée lors de la \#ManifPourTous. Précision, elle a déclaré avoir 9 enfants...

Certains tweets inventent de mini dialogues, sérieux ou humoristiques, entre deux locuteurs jugés représentatifs du camp adverse. C'est une sorte de pastiche qui imite le style présumé de l'adversaire (ici, celui d'un homosexuel ou celui d'un catholique bourgeois de droite), pour dévoiler la bassesse de ses arguments.

87. Fred \& Jo, vs êtes homosexuels : pourquoi préférez la GPA à l'adoption?- Avec l'adoption, le risque de tomber sur 1 enfant noir est élevé.

88. " Maman on va ou? "'" Manifester contre le bonheur des gens mon chéri " \#ManifDeLaHonte

\subsection{Négation polémique}

Dans un contexte polémique, la négation donne à voir un contre-discours, tout en s'employant en même temps à le combattre. Ses trois modalités particulières, les réfutations par négation, par retournement et par dénégation, ont pour point commun qu' «elles repoussent un discours dont simultanément elles portent l'inscription » (Krieg Planque 2012 : 210). Ces procédés apparaissent souvent dans des énoncés porteurs de contre-analyses.

La réfutation par négation présente une structure ordinaire « $\mathrm{X}$ n’est pas $\mathrm{P}$ », par laquelle elle réfute une affirmation qui est ou aurait été faite par l’adversaire.

89. L'humain n'est pas une marchandise : Ni PMA, ni GPA ! \#5oct \#ManifPourTous

90. L'humain n'est pas un objet! Pour ces pauvres femmes qui seront exploitées et risqueront la mort pour le désir de qq riches... NON À LA \#GPA

91. \#LMPT L'enfant n'est pas un bien de consommation au profit de qq'un \#GPA \#PMA jamais

92. \#enfant \# droit je cite: "l'enfant n'est pas un droit" manifestants de la manif' pour tous !

La réfutation par retournement obéit au schéma « c’est $\mathrm{X}$ que $\mathrm{P}$ et ce n'est pas Y que $\mathrm{P}$ » comportant une focalisation (une forme d'emphase).

93. C'est Adam et Eve, pas Adam et Adam. \#ManifPourTous

94. contre le mariage homosexuel Dieu a crée Adam \& Eve pas Benoit et Thomas !

La réfutation par dénégation quant à elle peut être formulée comme suit : « ce n’est pas Y que $\mathrm{P}$ mais $\mathrm{X}$ que $\mathrm{P}$ », où Y est soumis en même temps à la focalisation et à la négation.

95. C'est pas l'homosexualité qui est une maladie mais plutôt l'homophobie. \#ManifDeLaHonte

96. L'homosexualité n'est pas un choix. L'homophobie, en est un. \#ManifDeLaHonte 
97. La GPA contrairement au mariage n'est pas l'accomplissement d'un combat pour l'égalité, c'est juste l'extension de la sphère marchande.

98. Contrairement à l'intolérance, l'amour n'est pas dangereux. \#mariagegay \#ouiaumariagegay

Ce qui est frappant dans les tweets cités plus haut, c’est que la négation polémique porte rarement sur des propos effectivement tenus. L'assertion ainsi rejetée est sensée synthétiser la logique de l'adversaire, en en révélant l'aspect intolérable, dangereux ou absurde.

\subsection{Reformulation interdiscursive polémique}

Dans une interaction conflictuelle, un mode particulier de reprise de discours consiste en une reformulation polémique. D'un point de vue argumentatif, cette opération permet au locuteur de remettre en question une première saisie d'un état de chose et d'en proposer une autre (Steuckardt 2007 : 4). D'un point de vue pragmatique, une reformulation interdiscursive polémique constitue un procédé de rectification ou de dévoilement.

Parmi les marques formelles qui permettent de signaler l'abandon du segment reformulé au profit du segment reformulant, il y a en fait, en réalité, en clair, en somme, plutôt... Selon le cas, l'énoncé comportera la séquence complète (X, en fait/en réalité c'est $\mathrm{Y}$ ), ou seulement le segment reformulant (en fait/réalité c'est Y). Les marqueurs en fait et en réalité présentent la première assertion comme non conforme à la réalité, à l'inverse de la seconde. Cette première assertion n'est pas nécessairement (complètement) exprimée, elle peut être déduite de la seconde. C’est le cas de la majorité des tweets, qui reformulent polémiquement des énoncés lus ou entendus quelque part. Un motif récurrent employé dans nos corpus de tweets semble aller dans le même sens : «ce qui est ... c'est ... ». Il permet de réfuter un argument implicite, car présent et saillant dans la mémoire des débatteurs, par exemple celui qui a été exprimé sur Twitter ou vu sur des pancartes des manifestants.

99. En réalité, la \#ManifPourTous n'a qu'une volonté : imposer sa religion à la France. Ce temps là est fini, et heureusement.

100.Ça se dit cathos mais en fait vous êtes juste...des abrutis! \#ManifDeLaHonte \#PasEnMonNom

101.Clap clap clap"@XY PMA, GPA, "théorie du genre"... en fait, la Manif pour tous, c'est SOS fantasmes."

102.Ce qui est contre nature c'est de se battre contre le droit des autres. \#ManifPourTous \#ManifDeLaHonte

103. Ce qui est contre nature, c'est d'aller a l'encontre des droits d'une personne en fonction de son orientation sexuelle! \#ManifDeLaHonte

\subsection{Détournement}

La reprise d'un discours peut s'accompagner d'une transformation délibérée. Il peut s’agir aussi bien d'un défigement d'une formule culturellement stabilisée que d'un détournement immédiat, opéré à partir d'un énoncé ayant vu jour au cours de l’interaction.

Tout énoncé faisant partie du patrimoine culturel commun, et plus généralement tout contenu qui relève d'un préconstruit, est susceptible d’être repris et recyclé, par exemple défigé, aussi bien sur un mode plaisant qu'ironique. A l'évidence, l'existence d'un rapport de sens avec le sujet traité augmentera l'impact de la formule trouvée. Une référence biblique, un titre de film ou une phrase clef issue d'un jeu populaire, par exemple, auront d'autant plus de chances d'être détournés qu'ils renvoient à l'origine au domaine concerné par le débat (ici, la famille). Mais inversement, adopter un style ou un genre de discours fortement connoté et décalé par rapport au sujet traité (par exemple, un contrat de vente en viager) peut servir à frapper l'imagination en pointant des risques insoupçonnés (celui d'une dérive marchande...).

104. C'est Adam et Eve, pas Adam et Adam. \#ManifPourTous

105.dans la famille SousLaTerrasse, je voudrais le père-pioche !\#7FamilleDeLigonnès 
106.Dans la famille Pourtous je voudrais le père !- Lequel ?\#mariagepourtous

107.Deux hommes et un couffin : un remarquable document @13h15 sur la \#GPA ! Un grd bravo ! Et un grd bravo aux papas!

108. La \#GPA est un viager gestationel où la mère porteuse n'a que la nue propriété de son enfant jusqu'à ce que s'exerce l'usufruit du payeur.

109.La \#GPA, c'est une lubie de la bourgeoisie "progressisto-libertaire" au service du grand capital. Objectif : créer un marché de l'enfant.

Différents modes de déformation et de défigement sont à l'œuvre dans des interactions à caractère polémique. Formellement, la transformation peut consister en une fusion, une substitution, une réduction, un déplacement, une expansion, une réduction, pour reprendre la typologie de Villers (2010). Avec \#cestlaluttenuptiale nous sommes en présence d'une particitation militante au sens de Maingueneau (2004), obtenue par substitution (finale $\rightarrow$ nuptiale). La substitution de syllabe (comète $\rightarrow$ gamète), de morphème (plan Vigipirate $\rightarrow$ Vichypirate ; égocentriques $\rightarrow$ égaux sans triques) ou de mot (deux $\rightarrow$ trois) avec une ressemblance formelle ou catégorielle optimale et des relations de sens aisément interprétables fait partie des mécanismes transformationnels les plus courants.

110.Envisager la GPA, c'est faire des plans sur la gamète.

111.les manifestants contre le \#mariagepourtous ont réussi : depuis hier, le plan Vichypirate est ouvert

112.Ceux qui défilent à la manif pour tous veulent que les hommes soient "égaux sans triques"

La question de l'égalité devant la loi a amené plusieurs participants au débat sur la procréation à reprendre dans leur argumentaire la devise républicaine "liberté, égalité, fraternité" ou l'article 1 de la Déclaration des Droits de l'Homme et du Citoyen «Les hommes naissent et demeurent libres et égaux en droits ». Par un simple ajout d'une expansion, il s'agit de dénoncer sur un mode ironique l'existence d'exceptions dans l'application de ces principes. Rappeler la ségrégation raciale ou les politiques de Vichy, par exemple en détournant des phrases qui en décrivent les manifestations, va dans le même sens.

113.les hommes naissent et demeurent libres et égaux en droits, sauf s'ils sont homosexuels, autant pour moi \#ManifDeLaHonte

114."Liberté, Egalité, Fraternité", mais que pour les blancs hétéro catholiques c'est ça ? \#ManifDeLaHonte

115.@LaManifPourTous Slogan\#ManifPourTous :"égaux mais différents". Euh, demain les homos s'assoient au fond du bus?\#segretariato \#ManifDeLaHonte

116.Alerte Vichypirate en cours \#Civitas \#manifpourtous

Le deuxième procédé qui mérite l'attention est le détournement des sigles. Cette pratique consiste à substituer d'autres mots commençant par les mêmes lettres à ceux qui composent le sigle original développé. Comme le fait remarquer Renard (2011 : 1), cette créativité langagière prétend le plus souvent à l'humour, mais elle se donne aussi pour objectif de dénoncer la « face cachée » de l’objet désigné par le sigle. L'auteur identifie quatre types de détournement selon leur finalité dominante : ludique, injurieux, antithétique et dévoilant.

Le détournement des sigles (GPA, PMA) attesté dans le corpus FIL possède une teneur polémique, sans véhiculer systématiquement une attitude hostile aux réalités ainsi désignées.

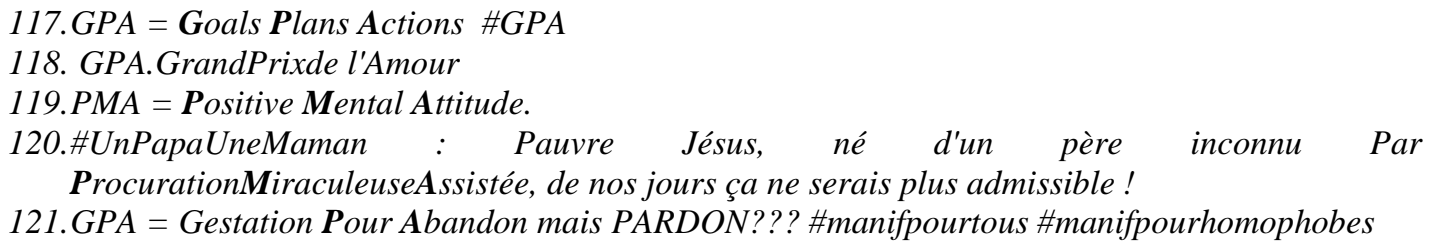

Le détournement des définitions, et notamment celles que l'un des protagonistes donne aux entités qu'il entend protéger ("La famille c'est un père et une mère"), correspond globalement à la même logique. La dérision et le dévoilement des faces sombres des familles traditionnelles sont clairement présents dans les 
tweets ci-après. Certains mots fonctionnent comme des rappels mémoriels à des événements socialement marquants (congélateur).

122. La famille idéale c'est :un papas une maman, un cheval un mouton

123.Un papa + une maitresse + une maman + un curé + 12 enfants + une Renault Espace = une famille normale \#manifpourtous

124.\#ManifPourTous un papa, une maman. comme Jésus. euh... non, lui c'est pas un bon exemple. enfin si mais là non, mais euh... oh merde.

125.? Un grand frère + Un Papa, y a pas mieux pour un enfant ! ? \#TeamDeVilliers \#ManifDeLaHonte \#ManifPourTous

126.? Une pelle + Une terrasse ! Y a pas mieux pour des enfants ? \#TeamLigonnès \#ManifDeLaHonte \#ManifPourTous

127. Une famille c'est un papa, une maman, des enfants et un congélateur.

Les cas de détournement immédiat de mots-dièse sont nombreux. On constate une forte créativité qui amène à reprendre (et à travestir) certains mots-dièse devenus populaires, pour polémiquer ou au contraire pour soutenir un message. Ainsi, \#ManifPourTous a donné entre autres \#manifPloufPlouf, \#UnHommeUneFemme a inspiré \#UnMixerUneCafetière, \#UnConUneConne etc. Le passage de \#manifpourtous à \#manifpourpersonne (ou encore à \#manifpourhomophobes, \#manifcontreautrui...) correspond à un détournement antithétique, par « retournement », pour faire signifier à ce deuxième terme le contraire de ce que signifie ou évoque le premier.

128.Essayez donc d'être heureux avant de passer votre temps nous rendre malheureux. \#ManifPourTous \#ManifPourPersonne

L'expression Xpourtous, engendrée à partir de la formule mariage pour tous (devenue en mot-dièse \#mariagepourtous, avec des variantes \#MariagePourTous...) prend dans le corpus MAR 230 formes différentes (\#divorcepourtous, \#adoptionpourtous...) et apparaît dans 38000 occurrences. Le corpus FIL fait apparaître une orientation spécifique privilégiant, pour ce qui concerne les partisans de la PMA et la GPA, l'usage des mots-dièse qui renvoient à l'égalité (\#droitspourtous) et aux émotions ou attitudes sociales positives (\#amourpourtous, \#respectdetous, \#espoirpourtous).

129.Faudrait peut-être se reveiller, on est en 2014 et la famille à déjà bien évolué !! \#droitspourtous \#manifdelahonte

130.France. Octobre 2014. Encore une occasion de perdre espoir en la nature humaine. \#ManifDeLaHonte \#droitalafamillepourtous

131.Quand la \#ManifPourTous se rendra compte qu'elle n'est pas représentative des français? \#egalitepourtous

132.\#NotInMyName \#amourpourtous \#ManifDeLaHonte

133.Il est ou l'\#espoirpourtous, le \#respectdetous ?! \#ManifDeLaHonte

\section{Conclusion}

Les réseaux sociaux constituent aujourd'hui une arène privilégiée d’échanges sur les grandes questions ou controverses d'intérêt public, qu'elles soient d’ordre politique, sociétal, religieux ou économique. Collecter et analyser les traces verbales de ces controverses constitue un enjeu et une opportunité scientifique intéressante non seulement pour les sciences sociales et politiques (traçabilité du social) et l'analyse du discours (rhétorique de la controverse), mais aussi et de plus en plus pour le traitement automatique des langues et les linguistiques de corpus (veille et fouille d'opinions, veille sociale...). Une telle finalité nécessite des apports convergents, permettant de tenir compte (i) des caractéristiques spécifiques aux univers discursifs à support numérique, (ii) des propriétés linguistiques des discours à caractère polémique, sans oublier (iii) la dimension sociétale des processus conflictuels qui engendrent de tels discours. Un important travail de systématisation, de modélisation et de construction de ressources associées (linguistiques et informatiques) reste encore à accomplir dans cette perspective, en établissant des ponts entre les différentes disciplines concernées. 
Le travail que nous venons de présenter entend contribuer à la description de la matérialité linguistique des controverses sociétales. Mené sur des productions verbales issues d’un dispositif numérique de microblogage, il éclaire également le fonctionnement des interactions agonistiques telles qu'elles se manifestent sur Twitter, outil de communication devenu incontournable et omniprésent. S’il est manifeste, comme le dit Paveau (2015), que « la dimension technique configure les scripturalités », il est tout aussi vrai que les procédés discursifs qui y sont employés ne s'écartent pas fortement de ceux que l'on atteste dans des conditions de communication plus classiques. Notre réflexion n'implique donc nullement une coupure avec une linguistique plus « traditionnelle ». L'originalité vient plutôt du caractère réellement pluridisciplinaire de la démarche employée.

Les différents procédés linguistiques que l’on atteste dans des discours polémiques sont porteurs de contenus spécifiques. L’identification des configurations particulières de ces procédés peut permettre des lectures fines et ciblées des discours en question. Dans le registre que nous venons d'explorer, à savoir celui de l'intersubjectivité et l'interdiscursivité, nous pouvons en citer plusieurs. Quel est le degré d'interaction entre débatteurs ? Restent-ils sourds aux opinions adverses ou au contraire les reprennent-ils pour les discuter ? Quels sont les arguments les plus contestés ? Et quels contre-arguments leur sont opposés ? Quelles sont les « armes » employées face à l'adversaire ? Est-il visé pour lui-même ou pour ses opinions ? Dans quel registre se placent préférentiellement les débatteurs : distancié ou émotionnel ? Quelle est la culture de la controverse des différents protagonistes (maitrise de soi, maitrise de ses affects, respect de l'interlocuteur...) ? Quelles représentations stéréotypées « collent à la peau » des différentes catégories d'acteurs concernés par la controverse ? En somme, en ressort également une certaine image de la société, avec ses fondamentaux, ses préoccupations et ses aspirations, ses fragilités et ses fractures.

\section{Références bibliographiques}

Amossy, R., Burger, M. éds. (2011). Polémiques médiatiques et journalistiques. Le discours polémique en questions, Semen, 31.

Amossy, R. (2014). Apologie de la polémique. Paris : PUF.

Angenot, M. (1982). La Parole pamphlétaire. Typologie des discours modernes. Paris : Payot.

Angenot, M. (2008). Le dialogue de sourd. Traité de rhétorique antilogique. Paris : Mille et une Nuits.

Charaudeau, P., Maingueneau, D. (2002), Dictionnaire d'analyse du discours, Paris : Seuil.

Chateauraynaud, F. (2011). Argumenter dans un champ de forces. Essai de balistique sociologique. Paris : Editions Petra.

Cervulle, M., Pailler, F. (2014). \#mariagepourtous : Twitter et la politique affective des hashtags, Revue française des sciences de l'information et de la communication [En ligne], 4 | 2014, mis en ligne le 01 janvier 2014, consulté le 13 mars 2016. URL : http://rfsic.revues.org/717.

Dascal, M. (1998). Types of polemics and types of polemical moves. Cmejrkova, S, J. Hoffmannova, O. Mulleriva \& J. Svetla (eds). Dialogue Analysis, VI, 1, Tubingen : Max Niemeyer, 15-33.

Dascal, M., Chang, H.-L. eds (2007). Traditions of Controversy. Amsterdam: John Benjamins

Desmons, E., Paveau, M.-A. éds (2008). Outrages, insultes, blasphèmes et injures : violences du langage et polices du discours. Paris : L'Harmattan.

Jackiewicz, A. (à paraître). Outls notionnels pour l'analyse des controverses, Questions de communication, "Questionner les humanités numériques, perspectives croisées ».

Jackiewicz, A., Vidak, M. (2014). Étude sur les mots-dièse. CMLF, Berlin, juillet 2014.

Julliard, V., Cervulle, M. (2013). "Différence des sexes" et controverses médiatiques : du débat sur la parité au “mariage pour tous” 1998-2013. Le Temps des médias, 21, 161-175.

Kerbrat-Orecchioni, C. (1980). La polémique et ses définitions. In Gelas N. et C. Kerbrat-Orecchioni (éds), Le discours polémique, 3-40. 
Kerbrat-Orecchioni, C. (1986). L'implicite. Paris : Armand Colin.

Kerbrat-Orecchioni, C. (2005). Le discours en interaction. Paris : Armand Colin.

Koren, R. (2011). De la rationalité et/ou de l'irrationalité des polémiqueurs : Certitudes et incertitudes, Semen [En ligne], 31 | 2011, mis en ligne le 01 avril 2011, consulté le 13 mars 2016. URL : http://semen.revues.org/9061

Krieg-Planque, A. (2012). Analyser les discours institutionnels. Paris : Armand Colin.

Laforest, M., Vincent, D. (2004). La qualification péjorative dans tous ses états. Langue française, 144, 59-81.

Lagorgette, D. (2012). Insulte, injure et diffamation : de la linguistique au code pénal ? Argumentation et Analyse du Discours [En ligne], 8|2012, mis en ligne le 15 avril 2012, Consulté le 13 mars 2016. URL : http://aad.revues.org/1312.

Latour, B. (2007). La cartographie des controverses. Technology Review, N. 0, 82-83.

Lemieux, C. (2007). À quoi sert l'analyse des controverses ? Mil neuf cent. 25, 191-212.

Maingueneau, D. (1983). Sémantique de la polémique. Lausanne : L’Age d’Homme.

Maingueneau, D. (2004). Hyperénonciateur et 'particitation'. Langages, 156, 111-127.

Moise, C., Auger, N., Fracchiolla, B., Schultz-Romain, Ch. éds (2008). La violence verbale. vol 1et 2, Espaces politiques et médiatiques, Paris : L'Harmattan.

Oger, C. (2012). La conflictualité en discours : le recours à l'injure dans les arènes publiques. Argumentation et Analyse du Discours [En ligne], 8 | 2012, mis en ligne le 15 avril 2012, Consulté le 13 mars 2016. URL : http://aad.revues.org/1297

Paveau, M.-A. (2015). Ce qui s’écrit dans les univers numériques. Itinéraires [En ligne], 2014-1 | 2015, mis en ligne le 12 janvier 2015, consulté le 13 mars 2016. URL : http://itineraires.revues.org/2313 ; DOI : 10.4000/itineraires.2313

Plantin, Ch. (1997). L’argumentation dans l'émotion. Pratiques, 96, 81-100.

Plantin, Ch. (2003). Des polémistes aux polémiqueurs. In Declercq G., Murat M., Dangel J., La parole polémique, H. Champion, 377- 408, <halshs-00425283>.

Plantin, Ch. (2011). Les bonnes raisons des émotions. Principes et méthode pour l'étude du discours émotionné. Bern : Peter Lang.

Renard, J.-B. (2011). Le détournement de sigles. Entre jeu de mots et expression contestataire. Mots. Les langages du politique [En ligne], 95 | 2011, mis en ligne le 01 mars 2013, consulté le 13 mars 2016. URL : http://mots.revues.org/20052

Rennes, J. (2007). Analyser une controverse. De la science politique à l'étude argumentative. In S. Bonnafous et M. Temmar (dir.), Analyse de discours et sciences humaines et sociales, Paris : Ophrys, 91-107.

Reynié, D. éd. (2012). Valeurs partagées. Paris : PUF.

Rosier, L. éd. (2012). Insulte, violence verbale, argumentation, Argumentation et Analyse du Discours [En ligne], 8 | 2012, mis en ligne le 14 avril 2012.

Scherer, K.R. (1984). On the nature and function of emotion: a component process approach. In K.R. Scherer and P. Ekman (Ed.), Approaches to emotion, Hillsdale, NJ: Erlbaum, 293-317.

Steuckardt, A. (2007). Usages polémiques de la reformulation. Recherches linguistiques, 29, 55-74.

Villers, D. (2010). Les modalités du détournement proverbial : entre contraintes et libertés, Modèles linguistiques [En ligne], 62 | 2010, mis en ligne le 25 janvier 2013, consulté le 13 mars 2016. URL : http://ml.revues.org/237

\footnotetext{
${ }^{1}$ L’adjectif polémique vient du grec polemikos, « relatif à la guerre ».
} 
${ }^{2}$ Nous empruntons ce terme à (Steuckardt 2007).

3 «En France, il semble bien en outre (en l'absence de toute étude précise sur la question) que l'on assiste aujourd'hui à une raréfaction notable de l'emploi des noms d'adresse : dans bien des situations communicatives, la salutation et le remerciement ne s'accompagnent plus automatiquement d'un nom d'adresse comme le recommandent grammaires et traité de savoir-vivre (en revanche, le nom d'adresse apparaît volontiers aux côté d'un reproche, d'une protestation ou d'une réclamation, c'est-à-dire qu'il a souvent une connotation polémique). » (C.K.O. : Adresse (terme d'-), dans Charaudeau et Maingueneau $2002: 32$ ) 